\title{
THE SPATIOTEMPORAL VARIATION OF HEAVY NO2 POLLUTION CENTER (HPC): A CASE STUDY IN THREE CHINESE URBAN AGGLOMERATIONS
}

\author{
Yuan $\mathrm{Gao}^{1}$, Jiayi $\mathrm{Li}^{1}$, Xin. Huang ${ }^{1,2, *}$ \\ ${ }^{1}$ School of Remote Sensing and Information Engineering, Wuhan University, 129 Luoyu Road, Wuhan 430079, P \\ R China- (boheecat, zjjerica, xhuang)@whu.edu.cn \\ ${ }^{2}$ State Key Laboratory of Information Engineering in Surveying, Mapping and Remote Sensing, Wuhan University, \\ Wuhan 430079, P R China
}

\section{Commission III, WG III/8}

KEYWORDS: Heavy Pollution, Urbanization Level, Meteorology, Urban Agglomeration, $\mathrm{NO}_{2}$.

\begin{abstract}
:
Air pollution episode, which are periods with excessive air pollutants, can cause a sharp increase in mortality and morbidity. Nitrogen oxides have an adverse impact on human health and the environment. Previous studies mainly focus on the time period, the frequency, and the duration during of heavy $\mathrm{NO}_{2}$ pollution, while ignored its spatial extent which is pivotal in providing early warning and prediction. In this study, we investigated the spatiotemporal variation of the heavy $\mathrm{NO}_{2}$ pollution extent (i.e., heavy pollution center), analyzed its association with meteorological condition and further predicted its distribution in the future. A case study in Jing-Jin-Ji (JJJ), Yangtze River Delta (YRD) and Pearl River Delta (PRD) urban agglomerations showed that the HPC exhibited evident seasonal (winter > summer) and inter-city (mega and medium cities> small cities) differences. In concretion analysis, the HPC areas were negatively correlated with temperature and precipitation, suggesting that dry and cold meteorological conditions were responsible for the severe $\mathrm{NO}_{2}$ pollution events. Trend analysis showed that the small and medium cities may serve as the HPC in the future. During the $2005-2016$, the medium and small cities in JJJ experience a more rapid increase in $\mathrm{NO}_{2}$ concentration in comparison to mega cities. Meanwhile, in YRD and PRD, a more rapid decrease was witnessed in the mega cities. The results of this study would provide support for early warning and prediction of heavy air pollutants and offer scientific insights for air pollution episode management.
\end{abstract}

\footnotetext{
* Corresponding author
} 


\section{INTRODUCTION}

Over the last few decades, ecosystems, the Earth's climate, and air quality have deteriorated sharply because of increasing human activities (Ebi and McGregor, 2008; IPCC Report2014; Seinfeld et al., 1998). As a major pollutant, nitrogen oxides $\left(\mathrm{NO}_{2}\right)$ is receiving growing attention due to its several adverse outcomes on human(Chiusolo et al., 2011; Samoli et al., 2006). According to the results of epidemiological studies, the accumulation of $\mathrm{NO}_{2}$ in the human body can damage the cells of the respiratory tract, cause inflammation of the respiratory tract, thereby inducing acute or chronic poisoning, and death(Brunekreef and Holgate, 2002). Emergency department visits as well as the mortality for respiratory disease increase sharply during the heavy pollution episodes, which are the periods with high levels of air pollutions(Brunekreef and Holgate, 2002). With knowledge of the extent of heavy pollution, warning signals can be issued to take timely responses and to reduce public exposure to air pollution. Therefore, to effectively control the $\mathrm{NO}_{2}$ concentration and to take emergency measures towards heavy pollution episode, it is necessary to analyze the spatiotemporal variation of the heavy pollution center (HPC).

Meteorological condition play a significant role in air quality through influencing the emissions, transport, chemical transformations, and wet or dry removal processes of air pollutants(Liu et al., 2017; Zhang et al., 2015; Zhou et al., 2012). According to previous studies, there is close association between the heavy pollution episodes and meteorological condition which is unfavorable to air pollution dispersion(Czarnecka and Nidzgorska-Lencewicz, 2011; Wang et al., 2014a). However, previous studies mainly focused on the influence of meteorology on the time period, the frequency, and the duration during of heavy pollution, while few studies have explored its effects on the extent.

Owing to the difference in population growth rate, the urban sprawl speed and controlling measures towards air pollutants, the $\mathrm{NO}_{2}$ pollution levels of cities with different urbanization level can experience diverse change process. On the one hand, the increase in population is associated with the rise in energy consumption which can generate large quantities of air pollutions(Lamsal et al., 2013; Schneider et al., 2015). On the other hand, urban areas with higher urban sprawl speed tend to have a less compact urban form, that is, longer distance between neighborhoods. As a result of longer distance, the per capita daily vehicle-miles traveled increase, leading to a higher emission of $\mathrm{NO}_{2}$ (Bechle et al., 2011; Bechle et al., 2017). At the same time, stringent emissions standards may encourage some industries to move to surrounding areas with looser standards. Therefore, it is still needs to be revealed whether the high pollution center moves from cities with high urbanization level to cities with low urbanization level.

Traditionally, $\mathrm{NO}_{2}$ was monitored by ground-level measurements, however, it is difficult to rely on these data to analyze the distribution of air pollutions due to the sparse density of air monitoring stations and the interference of local conditions(Jin and Holloway, 2015; Lamsal et al., 2013; Schneider et al., 2015). Satellite remote sensing now provides the opportunity to quantify the spatiotemporal variations of $\mathrm{NO}_{2}$ pollution at the global scale. Over the past decades, there are many studies utilizing the satellite remote sensing data to study the temporal trends of $\mathrm{NO}_{2}$ concentration of cities(Choi and Souri, 2015; Jin and Holloway, 2015; Jing et al., 2014; Mahajan et al., 2015; Schneider et al., 2015). However, most of these studies regard the cities as a whole and neglect the spatial distribution of $\mathrm{NO}_{2}$ concentration. Therefore, they can ignore the impact of the urbanization process on the location of air pollution center.

Urban agglomerations, that is, high-density areas composed of multiple cities, usually include multiple cities with different urbanization stages, economic levels, population density, and pollution control measures(Fang and Yu, 2017). As some of the most 
populous urban agglomerations in China, the Jing-Jin-Ji (JJJ), Yangtze River Delta (YRD) and Pearl River Delta (PRD) have experienced rapid urbanization and serious $\mathrm{NO}_{2}$ pollution(Jin and Holloway, 2015; Wang et al., 2014b). Therefore, they are ideal research objects for analyzing the transfer of air pollutants among cities.

The main aims of this study were: 1) to investigate the relationship between the meteorological conditions and the spatial variation of the HPC across three urban agglomerations; 2) to examine temporal variations of the HPC in three urban agglomerations for the period of 2005-2016; and 3) to predict the HPC of three urban agglomerations in the future. Our analysis was based on multi-source datasets including the Dutch Ozone monitoring instrument $\mathrm{NO}_{2}$ (DOMINO-V2), the NCEP/NCAR meteorological reanalysis data and census data from China Statistical Yearbooks Database (Yearbook, 2012). The HPC for each city was obtained by the Jenks natural breakpoint method and its association with meteorological condition was examined by the Pearson correlation analysis. The HPC of urban agglomeration was predict by the linear regression model and a bootstrap technique. The results of this study will help to deepen the understanding of the spatiotemporal variation of HPC, and will provide support for air pollution control.

\section{FEATURES OF THE HPC}

\subsection{Study Areas}

JJJ locates in northern China and covers an area of 217156 square kilometers (Yearbook, 2012). As the largest urban agglomeration in northern China, JJJ includes the economic zone of Beijing and Tianjin around the Bohai Sea. It is composed of 10 cities, including Beijing, Tianjin, Baoding, Shijiazhuang, Tangshan, Cangzhou, Langfang, Zhangjiakou, Chengde and Qinhuangdao. As China's political and cultural center region and international exchange center region, JJJ urban agglomeration has experienced a rapid process of urbanization after the reform.
The YRD urban agglomeration is located in the center of the south of the Yangtze River, where the Yangtze River enters the East China Sea. The Yangtze River Delta is one of the most populous areas on the planet, including Shanghai, one of the world's largest cities, with a population density of 2700 people per square kilometer(Yearbook, 2012).It covers an area of 99600 square kilometers (38500 square miles) and is composed of 16 cities, including Shanghai, Nanjing, Wuxi, Changzhou, Suzhou, Nantong, Yangzhou, Zhenjiang, Taizhou, Hangzhou, Ningbo, Jiaxing, Huzhou, Shaoxing, Zhoushan and Taizhou.

As one of the most populous urban areas in the world, the PRD has become the largest and most populous city in the world. It is located in the intersection of three rivers, covering an area of 39380 square kilometers. The PRD is composed of nine prefecture-level cities in Guangdong Province, namely Guangzhou City, Shenzhen City, Zhuhai City, Foshan City, Dongguan City, Zhongshan City, Jiangmen City and Huizhou City.

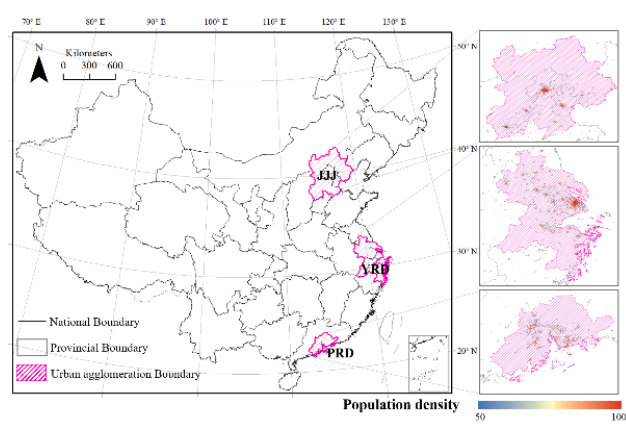

Figure 1. Study area

\subsection{Methods and Materials}

2.2.1 Detection of HPC: In order to analyze the spatial distribution of $\mathrm{NO}_{2}$ within urban agglomeration, the Jenks natural breakpoint method in Arcgis was utilized to divide the concentration of $\mathrm{NO}_{2}$ into four categories, namely, "high", "subhigh", "sublow" and "low" level(Peng et al., 2016). The Jenks natural breakpoint method divides the category by maximizing the inter-class variance and minimizing the intra-class variance. The level with the highest $\mathrm{NO}_{2}$ concentration is defined as Heavy Pollution Center (HPC).

$\mathrm{NO}_{2}$ concentration is obtained from Ozone monitoring 
instrument (OMI). OMI is a visible ultraviolet spectrometer on board NASA's Aura spacecraft. After TOMS, OMI records the total amount of several air pollutants and atmospheric parameters related to air pollution chemistry and climate(Ziemke et al., 2006). OMI can distinguish aerosol types such as smoke, dust and sulfate, measure standard pollutants such as $\mathrm{O}_{3}, \mathrm{NO}_{2}, \quad \mathrm{SO}_{2}$ and aerosols, and provide cloud pressure and coverage. As a wide-field imaging spectrometer, OMI has a cross-section angle of view of $114^{\circ}$ and can be measured in a global range within 1 day. Its spatial resolution is $13 \times 13 \mathrm{~km}$, which can be used to detect and track pollution sources at the urban scale(Ziemke et al., 2006). In this study, the tropospheric $\mathrm{NO}_{2}$ column concentration was collected from Royal Netherlands Meteorological Institute during 2005 - 2016(Boersma et al., 2010).

To analyze the spatial distribution of HPC, the cities of three urban agglomerations were divided into different level according to the population density growth rate. According to previous studies, population density can reflect the urbanization level of cities(Han et al., 2014). Considering that the population density can experience rapid variation over 12 years, the growth rate of population density, instead of the population density of a given year, was utilized as the indicator. Cities were categorize into three levels according to the population density growth rate provided by the China Statistical Yearbook (Yearbook, 2012): $\geq 10$ people per $\mathrm{km}^{2}$ (mega city, level 3); 3-5 people per $\mathrm{km}^{2}$ (medium city, level 2 ); $\leq 3$ people per $\mathrm{km}^{2}$ (small city, level 1).

\subsubsection{Association between Meteorological}

Condition and HPC: In order to analyze the influence of meteorological conditions on $\mathrm{NO}_{2}$ concentration, four meteorological conditions were considered in this study, including: 1) air temperature, 2) total rainfall, 3) planetary boundary layer height and 4) wind speed.

For the study period, the monthly mean total rainfall data at a $0.25^{\circ}$ spatial resolution were collected from the Tropical Rainfall Measuring Mission
(TRMM)(Council, 2006). The monthly mean planetary boundary layer height at 12:00 UTC (the closest to the OMI overpass time) was derived from the Modern-Era Retrospective analysis for Research and Applications, Version 2 (MERRA-2) dataset(Gelaro et al., 2017). The monthly mean air temperature and wind speed were obtained from the National Center for Environmental Prediction (NCEP)/National Center for Atmospheric Research (NCAR) 40-Year Reanalysis Project, with a spatial resolution of $2.5^{\circ}$ (Kalnay et al., 1996). Air temperature was obtained by averaging the monthly temperature over 2005-2016 at the pressure level from the surface to $360 \mathrm{hPa}$.

2.2.3The Prediction of HPC: To predict the location of $\mathrm{HPC}$, the trend of $\mathrm{NO}_{2}$ concentration was analyzed by using linear regression model and bootstrap sampling method (Choi and Souri, 2015; Gardiner et al., 2008; Jin and Holloway, 2015).

The existing research shows that this method can accurately estimate the trend of the data by considering the uncertainty in the trend, and has been widely used to study the changing trend of the long-term behavior of the atmospheric condition and air pollution(Choi and Souri, 2015; Gardiner et al., 2008; Jin and Holloway, 2015). In order to remove the influence of seasonal variation on trend estimation, Fourier term is combined to the multivariate linear regression model to simulate the periodicity of seasonal variation(Gardiner et al., 2008). Although thelinear regression model obtains robust results in trend analysis, it is still vulnerable to outliers. In order to complement the linear regression model, a bootstrap sampling method with 2000 iterations was performed. If and only if the confidence interval does not contain zero, the trend is considered to be significant.

$$
\begin{array}{r}
m(t)=A+B t+\sum_{n=1}^{4} C_{n} \cos (n 2 \pi t) \\
+\sum_{n=1}^{4} D_{n} \sin (n 2 \pi t) \\
+\varepsilon
\end{array}
$$


where $\quad m(t)=$ the monthly average $\mathrm{NO}_{2}$

$t=$ the month

$A=$ the mean of the first year

$B=$ the trend

$C_{n}=$ the coefficients of the fourier term

$D_{n}=$ the coefficients of the fourier term

$\varepsilon=$ the error term

\subsection{Results and Discussion}

\subsubsection{The Relationship between Meteorological}

Condition and HPC: Figure 2 shows the spatial distribution of $\mathrm{NO}_{2}$ concentration in four seasons within three urban agglomerations. The areas of HPC varied greatly among different seasons, which reach largest in the winter. The HPC area of JJJ, YRD and PRD were 95476.6, 73734 and $5671 \mathrm{~km} 2$, respectively. In summer, the area of HPC is the smallest. The HPC area of JJJ, YRD and PRD were 3214, 0 and $0 \mathrm{~km} 2$, respectively.

Negative correlations between the HPC areas and the air temperature were observed $(\mathrm{R}=0.53, \mathrm{P}<0.01)$, which indicates that the areas of HPC would decrease with an increase in air temperature. The higher temperature indicates higher $\mathrm{OH}$ concentration which promote the photolysis of $\mathrm{NO}_{2}$. Therefore, $\mathrm{NO}_{2}$ are confined more closely to the emitted source regions during the periods with high temperature. Significant negative correlation was capture between HPC areas and precipitation, suggesting the sedimentation and dilution effect of precipitation on $\mathrm{NO}_{2}$. A positive correlation is observed between $\mathrm{NO}_{2}$ and wind speed since stronger winds facilitate the dispersion of air pollutants from the emission source to surrounding areas. The PBLH correlates positively with the areas of HPC, since lower PBLH inhibit dispersion of air pollutants, leading to the increase in HPC areas.

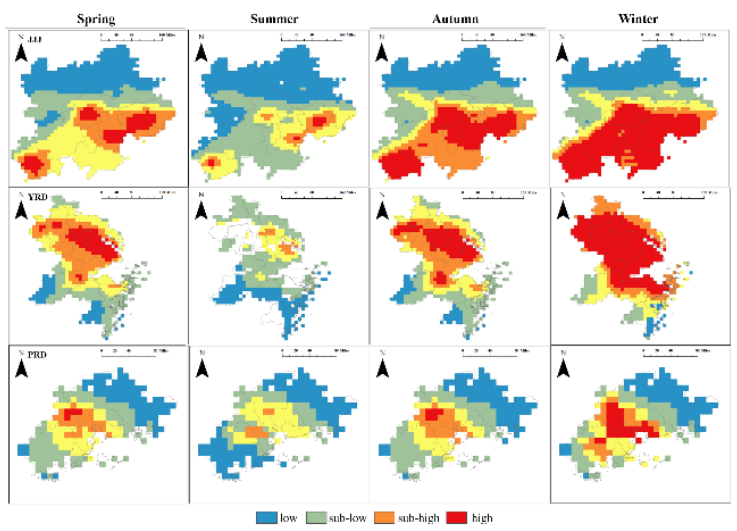

Figure 2. The spatial distribution of seasonal HPC in the JJJ, YRD and PRD

2.3.2 The Temporal Variation of HPC: The distribution and migration of HPC for the period of 2005-2016 was examined (Figure 3). In 2005, the HPC of three urban agglomerations was generally assembled around the mega-cities. A monocentric pattern was observed in PRD and YRD, while a multicentric distribution was captured in JJJ. However, in YRD, a small number of HPCs were evident in the medium cities, mainly distributed in YangZhou, NanJing and Hangzhou. In 2016, the mega cities and the medium cities in JJJ continue to be dominated by HPC; however, the area of HPC sharply increased from the 2005 level. In contrast, the areas of HPC experienced a dramatic decrease in PRD, due to the implement of stringent control measures.

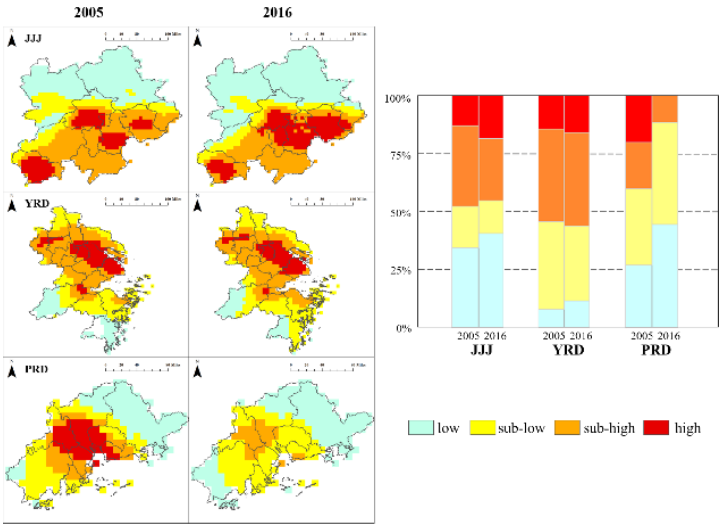

Figure 3. The spatial distribution and the proportions of HPC in JJJ, YRD and PRD in 2005 and 2016 
2.3.3 The Prediction of HPC : The trend of $\mathrm{NO}_{2}$ concentration in different city levels was further investigated (Figure 4). Across the three urban agglomerations, the trend of $\mathrm{NO}_{2}$ concentration of mega cities (i.e. level 3) is different from that of medium cities and small cities. In JJJ, cities at all levels experienced an increase in $\mathrm{NO}_{2}$ concentration, while the increase was more rapid in medium and small cities. In YRD and PRD, a decrease in $\mathrm{NO}_{2}$ concentration was witnessed in most cities, while the decline was relatively slower in medium and small cities. It is shown that the medium and small cities may serve as the HPC in the future.

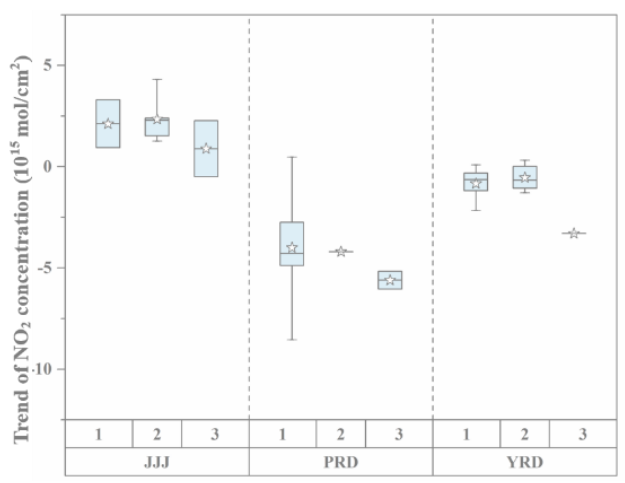

Figure 4. The trend of NO2 concentration during 2005-2016 in cities of different urbanization level

\section{CONCLUSION}

The heavy pollution center (HPC), which refers to the areas with high level $\mathrm{NO}_{2}$ pollution, was defined and investigated in this study. Based on the case study of three Chinese urban agglomerations during 2005-2016, we investigated the spatiotemporal variation of the HPC, analyzed its association with meteorological condition and further predicted its distribution in the future. The following conclusions were made:

1) The HPC during 2005 to 2016 was characterized by evident spatiotemporal heterogeneity, and the largest area were observed in winter, whereas the smallest areas was captured in winter. The correlation analyses showed that the HPC areas was negatively correlated with temperature and precipitation, indicating that dry and cold meteorological conditions were responsible for the severe $\mathrm{NO}_{2}$ pollution events. The HPC varied greatly among cities, and mega and medium cities tended to have larger areas than small cities.

2) From 2005 to 2016, the areas of HPC increased significantly in JJJ, especially in the medium cities, including the Tangshan and Langfang. In PRD, the HPC decreased sharply in all-leveled cities, suggesting the large scale success of air pollution control strategies targeted at $\mathrm{NO}_{2}$.

3) Inter-annually, the $\mathrm{NO}_{2}$ concentration increased significantly in JJJ, while the increasing rates of medium and small cities were nearly twice as high as those in mega cities. A significant decrease trend was observed in YRD and PRD, and the $\mathrm{NO}_{2}$ decrease more sharply in mega cities. Hence, the medium and small cities may serve as the HPC in the future.

\section{REFERENCES}

Ebi, K. L., and G. McGregor, 2008. Climate change, tropospheric ozone and particulate matter, and health impacts, Environmental health perspectives, 116(11), 1449-1455, doi:10.1289/ehp.11463.

IPCC Report 2014, C. C. Climate Change 2014: IPCC Report.

Seinfeld, J. H., S. N. Pandis, J. H. Seinfeld, and S. N. Pandis, 1998. Atmospheric chemistry and physics: from air pollution to climate change, 26-26 pp., Wiley.

Chiusolo, M., E. Cadum, M. Stafoggia, C. Galassi, G. Berti, A. Faustini, L. Bisanti, M. A. Vigotti, M. P. Dessì, and A. Cernigliaro, 2011. Short-term effects of nitrogen dioxide on mortality and susceptibility factors in 10 Italian cities: the EpiAir study, Environmental health perspectives, 119(9), 1233-1238.

Samoli, E., E. Aga, G. Touloumi, K. Nisiotis, B. 
Forsberg, A. Lefranc, J. Pekkanen, B. Wojtyniak, C. Schindler, and E. Niciu, 2006. Short-term effects of nitrogen dioxide on mortality: an analysis within the APHEA project, European Respiratory Journal, 27(6), $1129-1138$

Brunekreef, B., and S. T. Holgate, 2002. Air pollution and health, The lancet, 360(9341), 1233-1242.

Liu, T., et al, 2017. Attributions of meteorological and emission factors to the 2015 winter severe haze pollution episodes in China's Jing-Jin-Ji area, Atmos. Chem. Phys., 17(4), 2971-2980, doi:10.5194/acp-17-2971-2017.

Zhang, H., Y. Wang, J. Hu, Q. Ying, and X.-M. Hu, 2015. Relationships between meteorological parameters and criteria air pollutants in three megacities in China, Environmental Research, 140, 242-254,

doi:https://doi.org/10.1016/j.envres.2015.04.004.

Zhou, Y., D. Brunner, C. Hueglin, S. Henne, and J. Staehelin, 2012. Changes in OMI tropospheric NO2 columns over Europe from 2004 to 2009 and the influence of meteorological variability, Atmospheric Environment, 46, 482-495, doi:10.1016/j.atmosenv.2011.09.024.

Wang, Y., L. Yao, L. Wang, Z. Liu, D. Ji, G. Tang, J. Zhang, Y. Sun, B. Hu, and J. Xin, 2014a. Mechanism for the formation of the January 2013 heavy haze pollution episode over central and eastern China, Science China Earth Sciences, 57(1), 14-25.

Czarnecka, M., and J. Nidzgorska-Lencewicz, 2011. Impact of weather conditions on winter and summer air quality, International Agrophysics, 25(1), 7-12.

Lamsal, L. N., R. V. Martin, D. D. Parrish, and N. A. Krotkov, 2013. Scaling relationship for NO2 pollution and urban population size: a satellite perspective, Environmental science \& technology, 47(14), 7855-7861, doi:10.1021/es400744g.
Schneider, P., W. A. Lahoz, and R. van der A, 2015. Recent satellite-based trends of tropospheric nitrogen dioxide over large urban agglomerations worldwide, Atmospheric Chemistry and Physics, 15(3), 1205-1220, doi:10.5194/acp-15-1205-2015.

Bechle, M. J., D. B. Millet, and J. D. Marshall, 2017. Does Urban Form Affect Urban NO2? Satellite-Based Evidence for More than 1200 Cities, Environmental science \& technology, 51(21), 12707-12716, doi:10.1021/acs.est.7b01194.

Bechle, M. J., D. B. Millet, and J. D. Marshall, 2011. Effects of Income and Urban Form on Urban NO2: Global Evidence from Satellites, Environmental science \& technology, 45(11), 4914-4919, doi:10.1021/es103866b

Jin, X., and T. Holloway, 2015. Spatial and temporal variability of ozone sensitivity over China observed from the Ozone Monitoring Instrument, Journal of Geophysical Research: Atmospheres, 120(14), 7229-7246, doi:10.1002/2015jd023250.

Jing, P., Z. Lu, J. Xing, D. G. Streets, Q. Tan, T. O'Brien, and J. Kamberos, 2014. Response of the summertime ground-level ozone trend in the Chicago area to emission controls and temperature changes, 2005-2013, Atmospheric Environment, 99, 630-640, doi:10.1016/j.atmosenv.2014.10.035

Mahajan, A. S., I. De Smedt, M. S. Biswas, S. Ghude, S. Fadnavis, C. Roy, and M. van Roozendael, 2015. Inter-annual variations in satellite observations of nitrogen dioxide and formaldehyde over India, Atmospheric Environment, 116, 194-201, doi:10.1016/j.atmosenv.2015.06.004.

Choi, Y., and A. H. Souri, 2015. Seasonal behavior and long-term trends of tropospheric ozone, its precursors and chemical conditions over Iran: A view from space, Atmospheric Environment, 106, 232-240, doi:10.1016/j.atmosenv.2015.02.012. 
Fang, C., and D. Yu, 2017. Urban agglomeration: An evolving concept of an emerging phenomenon, Landscape and Urban Planning, 162, 126-136.

Wang, Y., Q. Ying, J. Hu, and H. Zhang, 2014b. Spatial and temporal variations of six criteria air pollutants in 31 provincial capital cities in China during 2013-2014, Environment international, 73, 413-422, doi:10.1016/j.envint.2014.08.016.

Yearbook, C. S., 2012. National Bureau of statistics of China, China Statistical Yearbook.

Peng, J., P. Xie, Y. Liu, and J. Ma, 2016. Urban thermal environment dynamics and associated landscape pattern factors: A case study in the Beijing metropolitan region, Remote Sensing of Environment, $173,145-155$

Ziemke, J. R., S. Chandra, B. N. Duncan, L. Froidevaux, P. K. Bhartia, P. F. Levelt, and J. W. Waters, 2006. Tropospheric ozone determined from Aura OMI and MLS: Evaluation of measurements and comparison with the Global Modeling Initiative's Chemical Transport Model, Journal of Geophysical Research, 111(D19), doi:10.1029/2006jd007089.

Boersma, K. F. F., R. J. Dirksen, D. Brunner, Y. Zhou, V. V. Huijnen, H. Eskes, J. P. Veefkind, Q. L. Kleipool, Dobber, and P. Stammes, 2010. An improved retrieval of tropospheric $\mathrm{NO}_{2}$ columns from the Ozone Monitoring Instrument, Advanced Materials.
Han, L., W. Zhou, W. Li, and L. Li, 2014. Impact of urbanization level on urban air quality: a case of fine particles $(\mathrm{PM}(2.5))$ in Chinese cities, Environmental pollution, 194 ,

163-170, doi:10.1016/j.envpol.2014.07.022.

Council, N. R., 2006. Assessment of the Benefits of Extending the Tropical Rainfall Measuring Mission: A Perspective from the Research and Operations Communities: Interim Report, National Academies Press.

Gelaro, R., W. Mccarty, M. J. Suárez, R. Todling, A. Molod, L. Takacs, C. A. Randles, A. Darmenov, M. G. Bosilovich, and R. Reichle, 2017. The Modern-Era Retrospective Analysis for Research and Applications, Version 2 (MERRA-2), Journal of Climate, 30(14).

Kalnay, E., M. Kanamitsu, R. Kistler, W. Collins, D. Deaven, L. Gandin, M. Iredell, S. Saha, G. White, and J. Woollen, 1996. The NCEP/NCAR 40-Year Reanalysis Project, Bulletin of the American Meteorological Society, 77(3), 437-472.

Gardiner, T., et al, 2008. Trend analysis of greenhouse gases over Europe measured by a network of ground-based remote FTIR instruments, Atmos. Chem. Phys., $\quad 8(22)$ 6719-6727, doi:10.5194/acp-8-6719-2008. 